\title{
Share Videos, Not Pills: A Content Analysis of Prescription Drug Videos on YouTube for Presence of Behavior Change Theory
}

\author{
Joshua H. West, Cameron Lister, Jayne A. Perry, Jessica Lyn Church, David L. Vance \\ Department of Health Science, Brigham Young University, Provo, USA \\ Email: josh.west@byu.edu
}

Received 3 April 2014; revised 7 May 2014; accepted 14 May 2014

Copyright (C) 2014 by authors and Scientific Research Publishing Inc.

This work is licensed under the Creative Commons Attribution International License (CC BY). http://creativecommons.org/licenses/by/4.0/

(c) (i) D Den Access

\begin{abstract}
Objective: To identify the extent to which YouTube.com videos targeting prescription drug abuse include health behavior theory in their content and design. Methods: This study used a content analysis of 209 YouTube.com videos. Coders used an established theory-based instrument to rate each video's inclusion of theoretical constructs from four prominent health behavior theories. Each YouTube.com video was coded for twenty-two theoretical items, which were summed to create a total theory score. Results: The mean theory score of videos was 3.26 out of a possible 22 . Among the top $10 \%$ of videos with highest theory scores, social cognitive theory constructs were coded most often and transtheoretical constructs were coded least often. Conclusion: It is not unexpected that YouTube.com videos are largely void of theoretical content since these videos are made by a variety of organizations and sources that may not be trained in the application of health behavior theories. There is an opportunity for the organizations that create these videos to collaborate with health and behavior change experts in creating videos with more theoretical constructs, possibly resulting in better health outcomes.
\end{abstract}

\section{Keywords}

Behavioral Theories, Health Promotion, YouTube, Prescription Drug Abuse

\section{Introduction}

Prescription drug abuse (PDA) is the fastest-growing drug problem in the United States, and the Centers for Disease Control and Prevention (CDC) considers it as a critical public health issue [1]. PDA is the intentional use of a medication either, 1) without a prescription, 2) in a way other than as prescribed, or 3) for the expe- 
rience or feeling it causes. Whereas it is not a new problem, it is one that deserves renewed attention as rates of abuse and adverse effects continue to increase. About 1 in 9 youths aged 12 to 25 used prescription drugs nonmedically [2]. In 2009, nearly one-third of people aged 12 and over who used illicit drugs for the first time began by abusing a prescription drug [3]. Many prescription drug abusers, particularly teens, believe that these substances are safer than illicit drugs because they are prescribed by a healthcare professional and dispensed by a pharmacist [3]. PDA is likely to persist if these misperceptions and behaviors are not addressed with aggressive prevention efforts. Such efforts may involve elements of mass communication mediums that incorporate health behavior theory constructs, which have been shown to lead to behavior change [4]. Mass media communications, in the context of Internet-based resources, continue to show promise and are ideal for conveying positive health promotion messages [5].

The use of mass media campaigns as a means of changing health behavior is not uncommon. In recent years, however, the landscape of health-communication has begun changing rapidly with the emergence of online social media, which is still in the early adoption stages [6]. Fostering sustainable behavior change through affordable, community-based social marketing has been a challenge for traditional mediums of mass media campaigns [7]. With few exceptions, the limited success of behavior change efforts for traditional campaigns can be traced to the failure to properly apply health behavior theory to the development and implementation of effective interventions [8].

A proposed solution to the shortcomings of traditional media campaigns is the adoption of online mediums in which participatory social media plays a crucial role in health communication [9]. Social media combines the positive characteristics of both interpersonal and mass communication, making it a plausible utility for applying health behavior theory to promote behavior change [5]. Thus, online social networks have potential to serve as powerful vehicles to connect with social media users regarding drug use [10]. Addressing these inadequacies requires better analysis of effective public service announcement (PSA) production in online media outlets. The video-sharing website YouTube.com, for instance, is a social media site which presents a compelling platform for PSA distribution. YouTube.com allows users to publish and manage their own videos online, making it possible for amateur video producers to access a global audience. YouTube.com is one of the fastest growing sites in the United States, making it a potential resource for promoting beneficial and pro-social behaviors [11]. It is particularly salient as a potential platform for anti-drug PSAs since youths aged 12 - 17 comprise the largest demographic of viewers [12].

To address PDA prevention, health promotion may benefit from moving beyond less-effective methods of PSA-production and adapting to an online media-rich environment, incorporating social media sites such as YouTube.com. The purpose of this study was to evaluate the extent to which YouTube.com PSAs include health behavior theory in their prevention of prescription drug abuse.

\section{Methods}

\subsection{Study Design}

The study design involved a content analysis of health behavior theory in prescription drug abuse public service announcements (PSA) available on YouTube.com. The instrument and methods used for in this study were adapted from instruments developed by Doshi et al. (2003), which was created to evaluate the integration of health behavior theory in physical activity websites, and Cowan et al. (2012), which was created to evaluate the use of health behavior theory in physical activity iPhone apps [13] [14]. Additionally, two other studies evaluating health messages on YouTube and the inclusion of health behavior theory have been conducted using similar methodology [15] [16]. Two graduate research assistants trained in health behavior theory coded the videos to assess key constructs of 4 major theories of behavior change.

\subsection{Sample}

The study sample was selected from among YouTube.com videos in October of 2012. Search terms used to identify the study sample included "Prescription drug PSA, short", and 26 of the most commonly abused name-brand prescription drugs (i.e. "Adderall PSA, short") [17]. Researchers added the word "short" to the search query to exclude all videos over 4 minutes in length because videos shorter in length are more likely to be viewed in there entirety than longer videos. Furthermore, only videos that were in English language and produced by an organization were included in the study sample. Table 1 includes a list of the search terms and the 
Table 1. Search terms and results.

\begin{tabular}{ccc}
\hline & Search Terms & \\
\hline Terms Used & Number of YouTube Results & Number Included in Analysis \\
\hline Prescription drug PSA, short & 599 & 153 \\
Percocet PSA, short & 7 & 1 \\
Dexedrine, short & 179 & 7 \\
Adderall PSA, short & 37 & 6 \\
Ritalin PSA, short & 47 & 3 \\
Avinza, short & 18 & 3 \\
Codeine PSA, short & 8 & 2 \\
Oxycontin PSA, short & 50 & 15 \\
Percodan, short & 79 & 7 \\
Mebaral, short & 2 & 1 \\
Nembutal, short & 93 & 1 \\
Prosom, short & 74 & 2 \\
Tizanidine, short & 22 & 2 \\
Zanaflex, short & 50 & 1 \\
Actiq, short & 16 & 4 \\
Sublimaze & 5 & 1 \\
Total & 1521 & 209 \\
\hline
\end{tabular}

number of videos for each search term that met the inclusion criteria. After the inclusion criteria were applied, the final study sample consisted of 209 videos.

\subsection{Coding Procedure}

An Excel spreadsheet was created to capture the video title, video URL, view count, and length so as to maintain a static sample. Then, two graduate research assistants coded the videos independently after inter-rater reliability was established. Coders watched the sample videos one-by-one, using a theory-based instrument to analyze the video content. Researchers then entered the coded data into an electronic database.

\subsection{Measurement}

The instrument included constructs from four prominent health behavior theories, which included: the Health Belief Model (HBM), the Transtheoretical Model (TTM), the Theory of Planned Behavior (TPB), and Social Cognitive Theory (SCT). The instrument was comprised of a total of 22 theory-based items. Table 2 displays the theoretical variables included in the instrument. The instrument also included additional items that described the videos including the number of video views, the sponsoring organization (non-governmental organization, government organization, business, non-professional organization), original intended media (e.g. YouTube.com, television, radio), the age of the target audience (young adults, adults, professionals, general audience, other), race/ethnicity of characters in the videos, relationships in the videos (parent to child, friendship, etc.), overt message (out of reach/hidden, don't share, use only as prescribed, addiction recovery, other), tactics employed, and application of the precede-proceed model (predisposing factors, enabling factors, reinforcing factors).

\subsection{Analysis}

To verify the level of inter-rater reliability, the two coders independently coded a common $3 \%$ of the study videos. A Cohen's kappa coefficient was calculated to measure inter-rater agreement $(k=0.59)$. This level of agreement is considered high to moderate agreement, and is an acceptable level of inter-rater agreement [18]. Next, the sample was divided in half, with each half being independently coded by one of the coders. A Cohen's kappa coefficient was also calculated at the end of the coding on a common sample of $3 \%$ of the study videos ( $\mathrm{k}$ 
Table 2. Theory integration and variable measurement, $n=209$.

\begin{tabular}{|c|c|c|c|}
\hline Theory $^{\mathrm{a}}$ & Variable & Description & $\%$ \\
\hline $1,2,3,4$ & Knowledge & General information about PDA & 88.04 \\
\hline $1,2,3,4$ & Perceived benefits & $\begin{array}{l}\text { Information about the benefits of not engaging in } \\
\text { prescription drug abuse }\end{array}$ & 1.44 \\
\hline $1,2,4$ & Perceived barriers & $\begin{array}{l}\text { Addresses difficulties in trying to avoid PDA (e.g. peer } \\
\text { pressure, ease of accessibility) }\end{array}$ & 29.19 \\
\hline 1,3 & Perceived risks & $\begin{array}{l}\text { Information about PDA risks (e.g. addiction, illness, } \\
\text { death) }\end{array}$ & 77.51 \\
\hline $2,3,4$ & Self-efficacy & Mentions concept of confidence building & 0.96 \\
\hline 2,4 & Self-talk & $\begin{array}{l}\text { Examples of self-statements viewers can use in real-life } \\
\text { situations }\end{array}$ & 0.96 \\
\hline $2,3,4$ & Perceived social norms & Number of people who engage in PDA & 31.10 \\
\hline 2,4 & Self-monitoring & Instructions for self-monitoring & 0 \\
\hline 4 & Realistic goal setting & Discusses how to make achievable goals & 0 \\
\hline 2,4 & Stimulus-control & Cues and prompts identified & 0 \\
\hline 4 & Negative stimuli & Examples of PDA stimuli encountered by abusers & 0 \\
\hline 2 & Self-reward & Instructions for self-reward after PDA avoidance & 0 \\
\hline 2,4 & Social support & $\begin{array}{l}\text { Provides community source, social network (e.g. } \\
\text { community resources, local events, support groups) }\end{array}$ & 7.18 \\
\hline 4 & Negative social influence & Addresses topic of peer pressure & 0 \\
\hline 4 & Modeling/vicarious learning & Shows examples of non-drug-abusing role models & 4.31 \\
\hline 2,4 & Relapse prevention & $\begin{array}{l}\text { Provides ways to prevent engaging again for those trying } \\
\text { to quit }\end{array}$ & 0.96 \\
\hline 4 & Stress management & $\begin{array}{l}\text { Addresses skills such as relaxation techniques, } \\
\text { biofeedback, etc. }\end{array}$ & 0 \\
\hline 4 & Negative affect management & Mood management & 0 \\
\hline 2,4 & Skill building & Addresses how to gain skills & 11 \\
\hline $1,2,3$ & Emphasis on increasing knowledge & Links to other sources, referral sources & 62.20 \\
\hline 2 & Motivational readiness & Identifies how to find out if one is ready to change & 0.48 \\
\hline Total theory & & Mean (standard deviation) & $3.3(1.43)$ \\
\hline
\end{tabular}

${ }^{\mathrm{a}}$ Note: 1 = Health belief model, 2 = Transtheoretical model, 3 = Theory of planned behavior, 4 = Social cognitive theory.

$=0.63)$. There was no rater drift during the coding. Each YouTube.com video was coded for 22 theoretical items (Yes-1 No-0). A total theory score was calculated by summing the values from each theoretical variable. Descriptive statistics were calculated for each variable.

\section{Results}

Characteristics of the study videos are described in Table 3. Non-Governmental organizations posted the largest quantity of videos (38\%), followed by governmental organizations (27\%) and then non-professional organizations (20\%). Business represented the smallest portion of videos (15\%). Most videos targeted a general or unspecified audience, and the most common message was that prescription drugs should be used only as prescribed. Other descriptive information is included in Table 3.

The mean total theory score was $3.26(\mathrm{SD}=1.43$ ) out of a possible twenty-two. Table 4 presents the top ten percent of study videos according to total theory score. This table also displays these videos' use of each of the four health behavior theories included in the current study. The video with the highest total theory score, "Prescription Drug Abuse from Unison BHG", used 83\% of TPB constructs, 80\% of SCT, 36\% of HBM and 27\% of TTM. Among the top $10 \%$ of study videos in total theory score, SCT constructs were coded most often and TTM constructs were coded least often. 
Table 3. Descriptive information about YouTube.com study videos, $\mathrm{n}=209$.

\begin{tabular}{ccc}
\hline Variable & N $=$ XXX & $\%$ \\
\hline Organization & & \\
\hline Non-governmental organization (NGO) & 85 & 37.61 \\
Government organization & 60 & 26.55 \\
Business & 35 & 15.49 \\
Non-professional (school, contest, etc.) & 46 & 20.35 \\
\hline Intended audience of the video & & 17.76 \\
\hline Young adults & 38 & 27.10 \\
\hline Adults (parents/grandparents) & 58 & 0.47 \\
Professionals & 1 & 50.0 \\
General audience & 107 & 4.67 \\
Other & 10 & 28.79 \\
\hline Overt messages & & 6.23 \\
\hline Out of reach/hidden & 74 & 35.02 \\
Don't share & 16 & 5.06 \\
Use only as prescribed & 90 & 24.9 \\
Addiction recovery & 13 & 66.03 \\
\hline Other & 64 & 23.92 \\
\hline Precede-proceed model & & \\
\hline Predisposing factors & 50 & \\
Enabling factors & 138 & \\
Reinforcing factors & & \\
\hline
\end{tabular}

Table 4. Table type styles (table caption is indispensable).

\begin{tabular}{|c|c|c|c|c|}
\hline Video Title & HBM & TTM & SCT & ТPB \\
\hline Prescription Drug Abuse from Unison BHG & 0.36 & 0.27 & 0.8 & 0.83 \\
\hline PSA: Sheriff Brad Steub-Rx Drugs & 0.36 & 0.33 & 0.8 & 0.5 \\
\hline Be Aware. Don’t Share. Lock Your Meds & 0.36 & 0.2 & 0.8 & 0.67 \\
\hline Taking Action on Drug Disposal PSA & 0.29 & 0.27 & 0.6 & 0.5 \\
\hline Prescription Drug Abuse PSA & 0.43 & 0.33 & 0.6 & 0.67 \\
\hline PSA Prescription Abuse & 0.36 & 0.2 & 0.8 & 0.67 \\
\hline What Is the Percodan Treatment & 0.36 & 0.2 & 0.6 & 0.67 \\
\hline Rexburg Police Prescription Drug Takeback Program & 0.36 & 0.2 & 0.8 & 0.67 \\
\hline Connect the Pieces to Prevent Prescription Drug Abuse 60 sec PSA & 0.29 & 0.2 & 0.8 & 0.67 \\
\hline PSA Prescription and OTC Drug Abuse & 0.29 & 0.33 & 0.4 & 0.5 \\
\hline Attorney General Sam Olens’ PSA on Rx Drug Abuse & 0.36 & 0.2 & 0.8 & 0.67 \\
\hline NARCONON Georgia Prescription Drug Abuse among teenagers & 0.36 & 0.2 & 0.8 & 0.67 \\
\hline “What Does a Drug Dealer Look Like” & 0.36 & 0.2 & 0.8 & 0.67 \\
\hline Life’s Complicated Enough NIDA & 0.29 & 0.2 & 0.8 & 0.67 \\
\hline NARCONON Georgia Prescription Drug Abuse on the Rise & 0.29 & 0.13 & 0.8 & 0.67 \\
\hline Prescription Drug Abuse & 0.29 & 0.13 & 0.8 & 0.67 \\
\hline Rx-A Prescription Bottle Tells a Tale & 0.29 & 0.2 & 0.8 & 0.5 \\
\hline Household Hazardous Waste Drug PSA & 0.21 & 0.2 & 0.6 & 0.5 \\
\hline American Medicine Chest National PSA & 0.36 & 0.2 & 0.6 & 0.5 \\
\hline Prescription Drugs & 0.29 & 0.2 & 0.6 & 0.5 \\
\hline
\end{tabular}

Note: HBM = Health Belief Model; TTM = Transtheoretical Model; SCT = Social Cognitive Theory; TPB = Theory of Planned Behavior. 


\section{Discussion}

Theory-based interventions have been shown to be successful at changing health-related behavior [19], and may provide an effective framework for PSA-production, both on the individual and organizational level. The current study analyzed the level of health behavior theory inclusion in 209 PSAs found on YouTube.com. Results indicated that most of the sampled videos did not include health behavior theory, a finding that is not unexpected given that YouTube.com is universally accessible to potential PSA-creators, most of whom likely lack formal training in health behavior theory and behavior change. More surprising is the finding that this lack of theory persisted across all observed groups, including NGOs/voluntary organizations, government organizations, and businesses. This low level of theory incorporation in organization-made PSAs highlights the need for collaboration between health behavior-change experts (e.g., public health professionals and certified health education specialists) and organizational PSA creators.

Those videos that did employ elements of health behavior theory typically used a selection of 3 common strategies: 1) general information about PDA, 2) information about benefits of not engaging in PDA, and 3) information about risks of PDA. All other behavioral change strategies were employed by only $11 \%$ or fewer of the sampled videos. This result coincided with the findings of Doshi et al. (2003), who concluded that online physical activity websites provided adequate knowledge-based information and guidelines, but incorporated very few theory-based strategies [14]. This and other research has concluded that there is a need for websites to improve interaction and to better tailor assistance to the needs of users, since these methods are expected to have greater impact on behavior change [20]. This is consistent with the traditional approach of health education that largely focuses on the dissemination of health information. While the provision of education is an important part of supporting health behavior change, comprehensive approaches involving more intensive efforts should be included [21].

The absence of health behavior theory constructs in YouTube.com PDA PSAs presents an opportunity for health behavior specialists. The growing popularity of YouTube.com combined with the pressing need for theory-based PDA interventions should be incentive for health behavior change specialists to team with PSA developers in creating theory-based videos. Public health professionals can both provide expertise in health behavior change theory as well as advise video developers during the PSA developmental process. Video developers, meanwhile, might focus their efforts on creating videos with greater consumer appeal in order to boost the view counts of PSAs. A combination of these approaches might result in PSAs with greater effectiveness in deterring PDA. This study may also help clinicians, public health workers, and health educators make informed decisions about which YouTube PSAs to employ when attempting to combat PDA. These health professionals should take theory scores into account when selecting videos, and should have realistic expectations about the effectiveness of these videos in producing behavior change.

\section{Strengths and Limitations}

Although previous studies have assessed health-related YouTube.com videos for adherence to evidence-based practices, this is the first documented study to evaluate the presence of health behavior theory in YouTube.com videos. A major strength of this study is the use of a comprehensive evaluation tool adapted from Doshi et al. (2003). The results should be interpreted in the context of several limitations. First, it is common practice for YouTube.com users to copy videos and repost them to their own YouTube.com channels. As a result of this practice, a number of duplicate videos were found within the initial sample. For each set of duplicates, the video with the higher view count was retained and the others were removed from the study.

Furthermore, the broad search term "prescription drug PSA, short" might have been too general to return videos from all legitimate PDA campaigns. To address this challenge, a series of drug-specific YouTube.com searches were employed in order to include campaigns that might have been otherwise excluded by the general search. Lastly, the use of two video coders presented a limitation since the coding process is, to a certain extent, subjective. To address this challenge, the coders communicated throughout the study in order to adjudicate any discrepancies with respect to coding. To quantify the level of agreement, the coders performed two inter-rater reliability tests, one at the beginning of coding and one at the end. The concern regarding multiple coders was, in part, mitigated when the tests yielded Cohen's kappa coefficients in the acceptable ranges for studies of this nature. 


\section{Conclusion}

Most of the videos studied in this analysis utilized few theoretical constructs. However, there is a great potential for future partnership. Future steps may include the use of randomized control trials of YouTube.com videos to determine the efficacy of theory-based videos compared to videos that are not based on theory. Findings from this research, combined with the results of this study, would inform organizations that desire to create effective public service announcements. This also creates potential partnership opportunities between these organizations and health and behavior change experts, resulting in videos that incorporate theoretical constructs from established health behavior theories.

\section{References}

[1] CDC (2012) CDC Grand Rounds: Prescription Drug Overdoses_A US Epidemic. MMWR, 61, 10.

[2] SAMHSA (2011) Results from the 2010 NSDUH: Summary of National Findings. http://www.samhsa.gov/data/nsduh/2k10nsduh/2k10results.htm

[3] ONDCP (2011) A Response to the Epidemic to Prescription Drug Abuse. http://www.whitehouse.gov/sites/default/files/ondcp/Fact_Sheets/prescription_drug_abuse_fact_sheet_4-25-11.pdf

[4] Webb, T.L., Joseph, J., Yardley, L. and Michie, S. (2010) Using the Internet to Promote Health Behavior Change: A Systematic Review and Meta-Analysis of the Impact of Theoretical Basis, Use of Behavior Change Techniques, and Mode of Delivery on Efficacy. Journal of Medical Internet Research, 12, e4. http://dx.doi.org/10.2196/jmir.1376

[5] Cassell, M., Jackson, C. and Cheuvront, B. (1998) Health Communication on the Internet: An Effective Channel for Health Behavior Change? Journal of Health Communication, 3, 71-79. http://dx.doi.org/10.1080/108107398127517

[6] Thackeray, R., Neiger, B.L., Smith, A.K. and Van Wagenen, S.B. (2012) Adoption and Use of Social Media among Public Health Departments. BMC Public Health, 12, 242. http://dx.doi.org/10.1186/1471-2458-12-242

[7] McKenzie-Mohr, D. (2000) Fostering Sustainable Behavior through Community-Based Social Marketing. American Psychologist, 55, 531. http://dx.doi.org/10.1037/0003-066X.55.5.531

[8] Gielen, A.C. and Sleet, D. (2003) Application of Behavior-Change Theories and Methods to Injury Prevention. Epidemiolofic Reviews, 25, 65-76. http://dx.doi.org/10.1093/epirev/mxg004

[9] De Andrea, D.C. (2012) Participatory Social Media and the Evaluation of Online Behavior. Human Communication Research, 38, 510-528. http://dx.doi.org/10.1111/j.1468-2958.2012.01435.X

[10] Lord, S., Brevard, J. and Budman, S. (2011) Connecting to Young Adults: An Online Social Network Survey of Beliefs and Attitudes Associated with Prescription Opioid Misuse among College Students. Substance Use \& Misuse, 46, 66-76. http://dx.doi.org/10.3109/10826084.2011.521371

[11] Ahn, Y.-Y., Han, S., Kwak, H., Moon, S. and Jeong, H. (2007) Analysis of Topological Characteristics of Huge Online Social Networking Services. Proceedings of the 16th International Conference on World Wide Web, Banff, 8-12 May 2007, 835-844. http://dx.doi.org/10.1145/1242572.1242685

[12] Cheng, X., Dale, C. and Liu, J. (2007) Understanding the Characteristics of Internet Short Video Sharing: YouTube as a Case Study. IEEE Transactions on Multimedia, 15, 1184-1194. http://dx.doi.org/10.1109/TMM.2013.2265531

[13] Cowan, L.T., Van Wagenen, S.A., Brown, B.A., et al. (2013) Apps of Steel: Are Exercise Apps Providing Consumers with Realistic Expectations? A Content Analysis of Exercise Apps for Presence of Behavior Change Theory. Health Education \& Behavior, 40, 133-139. http://dx.doi.org/10.1177/1090198112452126

[14] Doshi, A., Patrick, K., Sallis, J.F. and Calfas, K. (2003) Evaluation of Physical Activity Web Sites for Use of Behavior Change Theories. Annals of Behavioral Medicine, 25, 105-111. http://dx.doi.org/10.1207/S15324796ABM2502_06

[15] Lister, C., Brutsch, E., Johnson, A., Boyer, C., Hall, P. and West, J.H. (2013) It Gets Better: A Content Analysis of Health Behavior Theory in Anti-Bullying YouTube Videos. International Journal of Health, 1, 17-24. http://dx.doi.org/10.14419/ijh.v1i2.1002

[16] Steadman, M., Chao, M.S., Strong, J.T., Maxwell, M. and West, J.H. (2014) CU L8ter: YouTube Distracted Driving PSAs Use of Behavior Change Theory. American Journal of Health Behavior, 38, 3-12. http://dx.doi.org/10.5993/AJHB.38.1.1

[17] Masters in Health Care (2011) 8 Most Abused Prescription Drugs. http://www.mastersinhealthcare.com/blog/2011/8-most-abused-prescription-drugs/

[18] Landis, J.R. and Koch, G.G. (1977) The Measurement of Observer Agreement for Categorical Data. Biometrics, 33, 159-174. http://dx.doi.org/10.2307/2529310

[19] Glanz, K., Rimer, B. and Viswanath, K. (2008) Health Behavior and Health Education: Theory, Research, and Practice. 
John Wiley \& Sons, Hoboken.

[20] Kreuter, M.W., Oswald, D.L., Bull, F.C. and Clark, E.M. (2000) Are Tailored Health Education Materials Always More Effective than Non-Tailored Materials? Health Education Research, 15, 305-315. http://dx.doi.org/10.1093/her/15.3.305

[21] Perkins, H. (2003) The Social Norms Approach to Preventing School and College Age Substance Abuse: A Handbook for Educators, Counselors, and Clinicians. Jossey-Bass, Hoboken. 